\title{
Pacific
}

Journal of

Mathematics

\section{A NOTE ON CONFORMAL RICCI FLOW}

Peng Lu, Jie Qing And Yu Zheng 


\title{
A NOTE ON CONFORMAL RICCI FLOW
}

\author{
Peng Lu, Jie Qing And Yu Zheng
}

\begin{abstract}
In this note we study the conformal Ricci flow that Arthur Fischer introduced in 2004. We use DeTurck's trick to rewrite the conformal Ricci flow as a strong parabolic-elliptic partial differential equation. Then we prove short-time existence for the conformal Ricci flow on compact manifolds as well as on asymptotically flat manifolds. We show that the Yamabe constant is monotonically increasing along conformal Ricci flow on compact manifolds. We also show that the conformal Ricci flow is the gradient flow for the ADM mass on asymptotically flat manifolds.
\end{abstract}

\section{Introduction}

Suppose that $M^{m}$ is a smooth $m$-dimensional manifold and that $g_{0}$ is a Riemannian metric on $M$ of constant scalar curvature $s_{0}$. All manifolds in this note are assumed to have no boundary. The conformal Ricci flow on $M$ is defined as

$$
\begin{cases}\frac{\partial}{\partial t} g+2\left(\text { Ric }-\frac{s_{0}}{m} g\right)=-2 p g & \text { in } M \times(0, T), \\ s_{g(t)}=s_{0} & \text { in } M \times[0, T)\end{cases}
$$

for a family of metrics $g(t)$ with initial condition $g(0)=g_{0}$ and a family of functions $p=p(t)$ on $M \times[0, T)$, where $s_{g(t)}$ is the scalar curvature of the evolving metric $g(t)$. The conformal Ricci flow (1-1) was introduced by Arthur Fischer [2004] as a modified Ricci flow that preserves the constant scalar curvature of the evolving metrics. It is so named because of the role that conformal geometry plays in maintaining constant scalar curvature. It was shown in [Fischer 2004] that on compact manifolds the conformal Ricci flow is equivalent to

$$
\begin{cases}\frac{\partial}{\partial t} g+2\left(\text { Ric }-\frac{s_{0}}{m} g\right)=-2 p g & \text { in } M \times(0, T), \\ (m-1) \Delta p+s_{0} p=-\mid \text { Ric }-\left.\frac{s_{0}}{m} g\right|^{2} & \text { in } M \times[0, T),\end{cases}
$$

with the initial condition $g(0)=g_{0}$. Based on the fact that the conformal Ricci flow (1-2) is of parabolic-elliptic nature, analogous to Navier-Stokes equations, the

Jie Qing's research was partially supported by NSF grant number DMS-1005295 and CNSF grant number 10728103. Yu Zheng's research was partially surpported by CNSF grant number 11271132 . MSC2010: primary 53C25; secondary 58J05.

Keywords: conformal Ricci flow, short-time existence, asymptotically flat manifolds, ADM mass. 
function $p$ was named the conformal pressure function in [Fischer 2004]. Using the theory of dynamical systems on infinite-dimensional manifolds, Fischer [2004] proved that conformal Ricci flow exists, at least for a short time, on compact manifolds with scalar curvature $s_{0}<0$. He also observed that the Yamabe constant monotonically increases along conformal Ricci flow on compact manifolds of negative Yamabe type. Therefore one can hope that the conformal Ricci flow does a good job of constructing Einstein metrics, considering the behavior of the Hilbert-Einstein action on the space of Riemannian metrics.

In this paper we adopt DeTurck's trick [1983; 2003] to eliminate the degeneracy of (1-2) from the symmetry of diffeomorphisms and consider

$$
\left\{\begin{aligned}
\frac{\partial}{\partial t} g+2\left(\operatorname{Ric}-\frac{s_{0}}{m} g\right) & =-2 p g+\mathscr{L}_{W} g, \\
(m-1) \Delta p+s_{0} p & =-\mid \text { Ric }-\left.\frac{s_{0}}{m} g\right|^{2} .
\end{aligned}\right.
$$

This is DeTurck's conformal Ricci flow for an appropriately chosen vector field $W$ (cf. Equation (3-5)) with an initial metric $g(0)=g_{0}$ of constant scalar curvature $s_{0}$. Equation (1-3) is a strong parabolic-elliptic partial differential equation. We use the contractive mapping theorem to prove an isomorphism property for the linearized DeTurck conformal Ricci flow and then we use the implicit function theorem to prove short-time existence for the DeTurck conformal Ricci flow. From this we obtain short-time existence for the conformal Ricci flow based on the discussion in Section 3.1.

Theorem 1.1. Let $\left(M^{m}, g_{0}\right)$ be a compact Riemannian manifold of constant scalar curvature $s_{0}$ with no boundary. Suppose that the elliptic operator $(m-1) \Delta_{g_{0}}+s_{0}$ is invertible. Then there exists a small positive number $T$ such that the conformal Ricci flow $g(t)$ with the initial metric $g_{0}$ exists for $t \in[0, T]$.

This extends the existence result in [Fischer 2004] to include some compact manifolds with scalar curvature $s_{0}>0$. For parabolic Hölder spaces and the theory of linear and nonlinear parabolic equations used in the proof, we take references mostly from [Lunardi 1995]. We also extend the monotonicity of the Yamabe constant in [Fischer 2004] as follows:

Theorem 1.2. Let $\left(M^{m}, g_{0}\right)$ be a compact Riemannian manifold and let $g(t), t \in$ $[0, T)$, be the solution of conformal Ricci flow with $g(0)=g_{0}$. Suppose that $g_{0}$ is the only Yamabe metric in the conformal class $\left[g_{0}\right]$ with scalar curvature $s_{g_{0}}=s_{0}$ and that $(m-1) \Delta_{g_{0}}+s_{0}$ is invertible. Then there is $T_{0} \in(0, T]$ such that each metric $g(t), t \in\left[0, T_{0}\right)$, is a Yamabe metric and the Yamabe constant $Y[g(t)]$ is strictly increasing for $t \in\left[0, T_{0}\right)$ unless $g_{0}$ is an Einstein metric.

This theorem indicates that the conformal Ricci flow is somehow a better family of constant scalar curvature metrics than those obtained in [Koiso 1979]. 
On asymptotically flat manifolds we use weighted Hölder spaces defined in [Lee and Parker 1987], and define weighted parabolic Hölder spaces based on the similar ones in [Lunardi 1995; Oliynyk and Woolgar 2007].

Theorem 1.3. Let $\left(M^{m}, g_{0}\right)$ be a scalar flat and asymptotically flat manifold with $g_{0}-g_{e} \in C_{-\tau}^{4, \alpha}$, where $\alpha \in(0,1), \tau \in(0, m-2)$, and $g_{e}$ is the standard Euclidean metric. Then there exists a small positive number $T$ such that the conformal Ricci flow $g(t)$ from the initial metric $g_{0}$ exists for $t \in[0, T]$ and $g(t)-g_{e} \in$ $C_{-\tau}^{1,2+\alpha}([0, T] \times M)$.

It is easily seen that (1-1) and (1-2) are equivalent on asymptotically flat manifolds because of the uniqueness of bounded solutions to linear parabolic equations on such manifolds. The scalar flat assumption in Theorem 1.3 is less stringent than it looks. Thanks to Schoen and Yau [1979, Lemma 3.3 and Corollary 3.1] we know that one can always conformally deform an asymptotically flat metric with nonnegative scalar curvature into a scalar flat and asymptotically flat metric.

Conformal Ricci flow is the gradient flow for the ADM mass on asymptotically flat manifolds (see Definition 4.1) in the following sense:

Theorem 1.4. Let $g(t)$ be the conformal Ricci flow obtained in Theorem 1.3 for $\tau \in\left(\frac{m-2}{2}, m-2\right)$. Then

$$
\frac{d}{d t} \boldsymbol{m}(g(t))=-2 \int_{M}\left|\operatorname{Ric}_{g(t)}\right|^{2} d \operatorname{vol}_{g(t)} .
$$

In particular, the ADM mass $\boldsymbol{m}(g(t))$ is strictly decreasing under conformal Ricci flow, except when $g_{0}$ is a Euclidean metric.

As a quick application of Theorem 1.4, one can easily show the rigidity part of the celebrated positive mass theorem of Schoen and Yau [1979]. The monotonicity of the ADM mass along conformal Ricci flow is sharply in contrast to the invariance of the ADM mass along Ricci flow on asymptotically flat manifolds [Dai and Ma 2007; Oliynyk and Woolgar 2007].

The organization of the paper is as follows: In Section 2 we introduce the conformal Ricci flow and establish the monotonicity of the Yamabe constant on compact manifolds. In Section 3 we prove short-time existence of the conformal Ricci flow, both on compact manifolds and on asymptotically flat manifolds. In Section 4 we recall the definition of the ADM mass and show that conformal Ricci flow on asymptotically flat manifolds is the gradient flow for the ADM mass.

\section{Conformal Ricci flow}

In this section we first introduce the conformal Ricci flow and then calculate evolution equations for curvatures along conformal Ricci flow. We then discuss the 
monotonicity of Yamabe quotients and Yamabe constants along conformal Ricci flow.

2.1. Conformal Ricciflow. Suppose that $M^{m}$ is a smooth $m$-dimensional manifold and that $g_{0}$ is a Riemannian metric on $M$ with constant scalar curvature $s_{0}$. In [Fischer 2004], the conformal Ricci flow on $M$ is defined by (1-1) for a family of metrics $g(t)$ with initial condition $g(0)=g_{0}$ and a family of functions $p=p(t)$ on $M \times[0, T)$.

As shown in [Fischer 2004], the normalization condition $s_{g(t)}=s_{0}$ in (1-1) may be replaced by an elliptic equation and one can rewrite (1-1) as (1-2). The equivalence between (1-1) and (1-2) was proved in [Fischer 2004, Proposition 3.2 and 3.4] when $M$ is a compact manifold. Based on the evolution equation for scalar curvature, it is easily seen that (1-1) always implies (1-2). Equation (1-2) implies (1-1) when the solution to the linear heat equation is unique, which is true both in the compact and asymptotically flat cases that we consider in this paper.

One important issue for geometric PDEs is the scaling property. It is easy to see that for any constant $\lambda>0$, if $g$ and $p$ solve the conformal Ricci flow (1-2), then

$$
\left.\left.g_{\lambda}(\cdot, t)\right)=\lambda^{-2} g\left(\cdot, \lambda^{2} t\right)\right) \quad \text { and } \quad p_{\lambda}(\cdot, t)=\lambda^{2} p\left(\cdot, \lambda^{2} t\right)
$$

also solve the conformal Ricci flow.

2.2. Curvature evolution equations under conformal Ricci flow. To understand conformal Ricci flow one often needs to calculate how curvatures behave along it. The calculations are straightforward. Consider a general geometric flow

$$
\frac{\partial}{\partial t} g=-2 T
$$

We recall that the evolution equations for curvatures are (see [Chow et al. 2006; Besse 1987])

$$
\begin{aligned}
\frac{\partial}{\partial t} S & =2 \Delta \Theta-2 \nabla^{i} \nabla^{j} T_{i j}+2 R^{i j} T_{i j}, \\
\frac{\partial}{\partial t} R_{i j} & =\Delta T_{i j}-\nabla_{i} \nabla^{k} T_{k j}-\nabla_{j} \nabla^{k} T_{k i}+\nabla_{i} \nabla_{j} \Theta+2 R_{i k j l} T^{k l}-R_{i k} T_{j}^{k}-R_{j k} T_{i}^{k}, \\
\frac{\partial}{\partial t} R_{i k j l} & =\nabla_{i} \nabla_{j} T_{k l}-\nabla_{i} \nabla_{l} T_{k j}-\nabla_{k} \nabla_{j} T_{i l}+\nabla_{k} \nabla_{l} T_{i j}-R_{i k j m} T_{l}^{m}-R_{i k m l} T_{j}^{m},
\end{aligned}
$$

where $\Theta:=g^{i j} T_{i j}$. For the conformal Ricci flow, where

$$
T=\operatorname{Ric}-\frac{s_{0}}{m} g+p g \quad \text { and } \quad \Theta=s-s_{0}+m p,
$$


we calculate the evolution equations for curvatures under the first equation in (1-2) and get

$$
\begin{aligned}
\frac{\partial}{\partial t} s & =\Delta s+\frac{2 s_{0}}{m}\left(s-s_{0}\right)+2 p\left(s-s_{0}\right)+2(m-1) \Delta p+2 s_{0} p+2\left|\operatorname{Ric}-\frac{s_{0}}{m} g\right|^{2}, \\
\frac{\partial}{\partial t} R_{i j} & =\Delta R_{i j}+2 R_{i k j l} R^{k l}-2 R_{i k} R_{j}^{k}+(m-2) \nabla_{i} \nabla_{j} p+\Delta p g_{i j}, \\
\frac{\partial}{\partial t} \mathrm{Rm} & =\Delta \mathrm{Rm}+\mathrm{Rm} * \mathrm{Rm}+\operatorname{Ric} * \mathrm{Rm}+\frac{2 s_{0}}{m} \mathrm{Rm}-2 p \operatorname{Rm}+\widetilde{T}\left(\nabla^{2} p\right),
\end{aligned}
$$

where the operator $*$ stands for contractions of tensors, Rm is the Riemann curvature tensor, and

$$
\widetilde{T}\left(\nabla^{2} p\right)_{i k j l}=g_{k l} \nabla_{i} \nabla_{j} p-g_{k j} \nabla_{i} \nabla_{l} p-g_{i l} \nabla_{k} \nabla_{j} p+g_{i j} \nabla_{k} \nabla_{l} p .
$$

2.3. Yamabe constants under conformal Ricciflow. On compact manifolds along conformal Ricci flow, we may calculate that

$$
\begin{gathered}
\Theta=m p, \quad \frac{\partial}{\partial t} d \operatorname{vol}_{g(t)}=-m p d \operatorname{vol}_{g(t)}, \\
\frac{d}{d t} \operatorname{vol}(M)=-m \int_{M} p d \operatorname{vol}_{g}=\frac{m}{s_{0}} \int_{M}\left|\operatorname{Ric}-\frac{s_{0}}{m} g\right|^{2} d \operatorname{vol}_{g} .
\end{gathered}
$$

Given a compact Riemannian manifold $\left(M^{m}, h\right)$, the Yamabe quotient is defined as

$$
Q[h]:=\frac{\int_{M} s_{h} d \operatorname{vol}_{h}}{\operatorname{vol}_{h}(M)^{(m-2) / m}}
$$

and the Yamabe constant is defined as

$$
Y[h]=\inf _{h \in[h]} Q[h] .
$$

A Riemannian metric $h$ is said to be a Yamabe metric if and only if

$$
Q[h]=Y[h] .
$$

Thus from (2-3) we have this:

Proposition 2.1. Suppose that $g(t), t \in[0, T)$, is a solution to the conformal Ricci flow (1-2) on a compact manifold with scalar curvature $s_{g_{0}} \neq 0$. Then the Yamabe quotient $Q[g(t)]$ is strictly increasing unless $g_{0}$ is an Einstein metric.

Next we give a proof of Theorem 1.2 concerning the evolution of Yamabe constants along conformal Ricci flow. As observed in [Wang and Zheng 2011; Chang and Lu 2007; Anderson 2005; Koiso 1979], the Yamabe constant could in general behave rather irregularly among manifolds of positive Yamabe type. 
Proof of Theorem 1.2. We prove the result by contradiction. Assume there is a sequence $t_{i} \rightarrow 0^{+}$such that $g\left(t_{i}\right)$ are not Yamabe metrics. Let $\tilde{g}_{i}$ be a Yamabe metric in the conformal class $\left[g\left(t_{i}\right)\right]$ of the same volume as $g\left(t_{i}\right)$. By the compactness of the space of Yamabe metrics of fixed volume, $\tilde{g}_{i}$ converges to a Yamabe metric $g_{\infty} \in\left[g_{0}\right]$ (taking a subsequence if necessary). By the assumption that $g_{0}$ is the only Yamabe metric in $\left[g_{0}\right]$, we have $g_{\infty}=g_{0}$. That is to say that both $g\left(t_{i}\right)$ and $\tilde{g}_{i}$ converge to $g_{0}$. Since $(m-1) \Delta_{g_{0}}+s_{0}$ is assumed to be invertible, we can apply Koiso's decomposition theorem [1979, Corollary 2.9] in the set of metrics of the same volume as $g_{0}$ to conclude the following: In some small neighborhood of $g_{0}$, each metric can be written uniquely as the product of a metric of constant scalar curvature near $g_{0}$ and a function. Since $\tilde{g}_{i}$ and $g\left(t_{i}\right)$ are in the same conformal class, have the same volume and both have constant scalar curvature, we get a contradiction.

Note that similar arguments have been used in [Wang and Zheng 2011; Chang and Lu 2007; Anderson 2005; Koiso 1979].

\section{Short-time existence of conformal Ricci flow}

In this section we prove the short-time existence of the conformal Ricci flow, i.e., Theorem 1.1 and 1.3. The first step is to combine the two equations in the conformal Ricci flow into one evolution equation with one nonlocal term. More precisely, (1-2) can be written as

$$
\frac{\partial}{\partial t} g+2\left(\operatorname{Ric}-\frac{s_{0}}{m} g\right)=-2 \mathscr{P}(g) g \quad \text { on } M,
$$

where

$$
\mathscr{P}(g)=\left((m-1) \Delta+s_{0}\right)^{-1} \mid \text { Ric }-\left.\frac{s_{0}}{m} g\right|^{2},
$$

provided that $(m-1) \Delta_{g(t)}+s_{0}$ is invertible for all $t \in[0, T]$. The strategy to prove the short-time existence for the conformal Ricci flow is similar to the one used in [DeTurck 1983; 2003] to prove the short-time existence for the Ricci flow. We will first prove the short-time existence for DeTurck conformal Ricci flow written as

$$
\frac{\partial}{\partial t} g+2\left(\operatorname{Ric}-\frac{s_{0}}{m} g\right)=-2 \mathscr{P}(g) g+\mathscr{L}_{W} g \quad \text { on } M .
$$

To prove the short-time existence for (3-2) we calculate the linearization of the DeTurck conformal Ricci flow and apply an implicit function theorem.

3.1. DeTurck's trick. As a system of differential equations, the conformal Ricci flow is of parabolic-elliptic nature, similar to the Navier-Stokes equations. The significant difference between the conformal Ricci flow and the Navier-Stokes equations is that the conformal Ricci flow is a geometric flow. Hence we need to 
find ways to eliminate the degeneracy of the conformal Ricci flow arising from the symmetries of diffeomorphisms.

In this subsection we will follow the idea of the improved version [DeTurck 2003] of the approach to short-time existence for the Ricci flow in [DeTurck 1983] to get rid of the degeneracy of diffeomorphisms for conformal Ricci flow. Before introducing DeTurck's trick, we first recall the following operator $G$. Let $g$ be a Riemannian metric on $M^{m}$. The operator $G$ on symmetric 2-tensor $B$ is defined as

$$
G(B)=B-\frac{1}{2} \operatorname{Tr}_{g}(B) g .
$$

Also recall the divergence operator $\delta$

$$
(\delta B)_{i}:=\nabla^{j} B_{i j}: \Gamma\left(S^{2}(M)\right) \rightarrow \Gamma\left(T^{*} M\right)
$$

and its adjoint operator $\delta^{*}$

$$
\left(\delta^{*} \omega\right)_{i j}:=-\frac{1}{2}\left(\omega_{i, j}+\omega_{j, i}\right): \Gamma\left(T^{*} M\right) \rightarrow \Gamma\left(S^{2}(M)\right) .
$$

Note that if $X$ is the dual vector field of $\omega$, then $\delta^{*} \omega=-\mathscr{L}_{X} g$, where $\mathscr{L}_{X}$ denotes the Lie derivative in the $X$ direction.

According to DeTurck's improved version [2003] of his approach to the shorttime existence of Ricci flow [DeTurck 1983], we consider the following gauge-fixed conformal Ricci flow on $M$ :

$$
\left\{\begin{aligned}
\frac{\partial}{\partial t} g+2\left(\operatorname{Ric}-\frac{s_{0}}{m} g\right) & =-2 p g+2\left(\delta^{*}\left(\tilde{g}^{-1} \delta G(\tilde{g})\right)\right), \\
(m-1) \Delta p+s_{0} p & =-\mid \text { Ric }-\left.\frac{s_{0}}{m} g\right|^{2}
\end{aligned}\right.
$$

for a family of metrics $g(t)$ with $g(0)=g_{0}$ and a family of functions $p(t)$ on $M \times[0, T)$, where $\tilde{g}$ is any fixed metric on $M$.

Suppose that $g(t), t \in[0, T)$, solves (3-4). Then we consider the time-dependent vector field $W$

$$
W^{k}:=g^{i j}\left(\Gamma_{i j}^{k}[g]-\Gamma_{i j}^{k}[\tilde{g}]\right),
$$

where $\Gamma_{i j}^{k}$ are the Christoffel symbols of the corresponding metric. It turns out that (see [Hamilton 1995, Section 6; Shi 1989])

$$
2\left(\delta^{*}\left(\tilde{g}^{-1} \delta G(\tilde{g})\right)\right)=\mathscr{L}_{W} g .
$$

Hence we can rewrite (3-4) as

$$
\left\{\begin{aligned}
\frac{\partial}{\partial t} g+2\left(\operatorname{Ric}-\frac{s_{0}}{m} g\right) & =-2 p g+\mathscr{L}_{W} g \\
(m-1) \Delta p+s_{0} p & =-\left|\operatorname{Ric}-\frac{s_{0}}{m} g\right|^{2} .
\end{aligned}\right.
$$


The conformal Ricci flow (1-2) and the DeTurck conformal Ricci flow (3-6) are related to each other by coordinate changes in the following sense. Suppose that $\hat{g}(t)$ solves the DeTurck conformal Ricci flow (3-6) and $W$ is given as in (3-5). We consider the one-parameter family of diffeomorphisms $\varphi_{t}$ generated by $W$ on $M$, defined by

$$
\frac{\partial}{\partial t} \varphi_{t}(x)=-W\left(\varphi_{t}(x), t\right), \quad \varphi_{0}(x)=x,
$$

for some time period $[0, T)$.

Lemma 3.1. Let $(\hat{g}(t), \hat{p}(t)), t \in[0, T)$, be a solution to the DeTurck conformal Ricci flow (3-6) on the manifold $M^{m}$ with the initial metric $g_{0}$. Assume that the solution $\varphi_{t}(x)$ to (3-7) exists for $t \in[0, T)$. Let

$$
g(t):=\varphi_{t}^{*} \hat{g}(t) \quad \text { and } \quad p(t):=\hat{p}\left(\varphi_{t}(x), t\right) .
$$

Then $(g(t), p(t)), t \in[0, T)$, is a solution to the conformal Ricci flow (1-2) on the manifold $M$ with $g(0)=g_{0}$.

Proof. By using (3-6) we simply compute that (cf. [Chow et al. 2006, Section 2.6])

$$
\begin{aligned}
\frac{\partial}{\partial t} g(t) & =\varphi_{t}^{*}\left(\frac{\partial}{\partial t} \hat{g}(t)\right)+\left.\frac{\partial}{\partial s}\right|_{s=0}\left(\varphi_{t+s}^{*} \hat{g}(t)\right) \\
& =-2 \varphi_{t}^{*}\left(\operatorname{Ric}_{\hat{g}}-\frac{s_{0}}{m} \hat{g}+\hat{p} \hat{g}\right)+\varphi_{t}^{*}\left(\mathscr{L}_{W} \hat{g}\right)-\mathscr{L}_{\left(\varphi_{t}^{-1}\right)_{*} W}\left(\varphi_{t}^{*} \hat{g}\right) \\
& =-2\left(\operatorname{Ric}_{g}-\frac{s_{0}}{m} g+p g\right) .
\end{aligned}
$$

The second equation for $p$ in (1-2) is readily seen to hold, since the scalar curvature under both flows is kept constant as $s_{0}$.

This lemma is particularly important to us because it enables us to prove the short-time existence of the conformal Ricci flow by proving the short-time existence of the DeTurck conformal Ricci flow. The later will be shown to be a system of parabolic-elliptic equations (see Lemma 3.3).

On the other hand, suppose that $(g(t), p(t)), t \in[0, T)$, solves the conformal Ricci flow (1-2) on $M$ with initial metric $g_{0}$. Let $\tilde{g}$ be any fixed metric on $M$. We then consider the harmonic map flow

$$
\frac{\partial}{\partial t} \varphi_{t}=\Delta_{g(t), \tilde{g}} \varphi_{t}, \quad \varphi_{0}=\mathrm{Id}
$$

for $\varphi_{t}: M \rightarrow M$, where the nonlinear Laplacian in local coordinates is

$$
\left(\Delta_{g_{1}, g_{2}} f\right)^{\gamma}=\Delta_{g_{1}} f^{\gamma}+\Gamma_{\alpha \beta}^{\gamma}\left[g_{2}\right] \frac{\partial f^{\alpha}}{\partial x^{i}} \frac{\partial f^{\beta}}{\partial x^{j}} g_{1}^{i j} .
$$

The following lemma is useful for deriving the uniqueness of the conformal Ricci flow from the uniqueness of the DeTurck conformal Ricci flow. From it readily 
follows that the uniqueness of the conformal Ricci flow with a given initial metric holds at least on compact manifolds.

Lemma 3.2. Let $(g(t), p(t)), t \in[0, T)$, be a solution to the conformal Ricci flow (1-2) on the manifold $M^{m}$ with the initial metric $g_{0}$. Assume that the solution $\varphi_{t}: M \rightarrow M$ to the harmonic map flow (3-8) exists for $t \in[0, T)$. Let

$$
\hat{g}(t):=\left(\varphi_{t}^{-1}\right)^{*} g(t) \quad \text { and } \quad \hat{p}(x, t):=p\left(\varphi_{t}^{-1}(x), t\right) .
$$

Then $(\hat{g}(t), \hat{p}(t)), t \in[0, T)$, is a solution to the DeTurck conformal Ricci flow (3-6) on the manifold $M$ with the initial metric $g_{0}$.

Proof. This follows from a calculation like the one in the proof of Lemma 3.1, after identifying the vector field $W$ with $\Delta_{g(t), \tilde{g}} \varphi_{t}$ (see [Chow et al. 2006, p. 117]).

3.2. Linearization of DeTurck conformal Ricciflow. In this subsection we compute the linearization of the DeTurck conformal Ricci flow (3-2). To do so we set

$$
g_{\lambda}(t)=g(t)+\lambda h(t)
$$

for a family of symmetric 2-tensors $h(t)$ and for $\lambda \in(-\epsilon, \epsilon)$. We rewrite the DeTurck conformal Ricci flow as

$$
M(g(t))=\frac{\partial}{\partial t} g+2\left(\operatorname{Ric}-\frac{s_{0}}{m} g\right)+2 \mathscr{P}(g) g-\mathscr{L}_{W} g=: \frac{\partial}{\partial t} g-\mathscr{F}(g(t))=0
$$

and calculate

$$
\left.\frac{d}{d \lambda}\right|_{\lambda=0} M\left(g_{\lambda}\right)
$$

To compute the linearization of $\mathscr{P}$ we first calculate (cf. [Chow et al. 2006, Equation (S.5), p. 547])

$$
\left.\frac{d}{d \lambda}\right|_{\lambda=0} \Delta_{g_{\lambda}} \mathscr{P}\left(g_{\lambda}\right)=-h_{i j} \nabla^{i} \nabla^{j} \mathscr{P}-\frac{1}{2}\left(2 \nabla^{i} h_{i j}-\nabla_{j} h_{i}^{i}\right) \nabla^{j} \mathscr{P}+\Delta \mathscr{P}^{\prime},
$$

where $\mathscr{P}^{\prime}=\left.\frac{d}{d \lambda}\right|_{\lambda=o} \mathscr{P}\left(g_{\lambda}\right)$. Next we may calculate

$$
\left.\frac{d}{d \lambda}\right|_{\lambda=0}\left|\operatorname{Ric}_{g_{\lambda}}-\frac{s_{0}}{m} g_{\lambda}\right|^{2}
$$

using the linearization of Ricci curvature

$$
\begin{aligned}
\left.\frac{d}{d \lambda}\right|_{\lambda=0} 2 R_{i j}\left[g_{\lambda}\right]=-\Delta h_{i j}-2 R_{i k j l} h_{k l} & +R_{i k} h_{j}^{k}+R_{j k} h_{i}^{k} \\
& -\nabla_{i} \nabla_{j} h^{k}{ }_{k}+\nabla_{i} \nabla^{k} h_{k j}+\nabla_{j} \nabla^{k} h_{k i}
\end{aligned}
$$

(see [Chow et al. 2006, (2.31)]). In summary we have

$$
(m-1) \Delta \mathscr{P}^{\prime}+s_{0} \mathscr{P}^{\prime}-P_{1}^{i j k l} \nabla_{i} \nabla_{j} h_{k l}-P_{2}^{i j k} \nabla_{i} h_{j k}+P_{3}^{i j} h_{i j}=0,
$$


that is,

$$
\mathscr{P}^{\prime}=\left((m-1) \Delta+s_{0}\right)^{-1}\left(P_{1} * \nabla^{2} h+P_{2} * \nabla h+P_{3} * h\right),
$$

where $P_{1}, P_{2}, P_{3}$ are tensors that depend on curvature of $g(t)$ and up to second-order spatial derivatives of $p$.

In the calculation of the linearization of $M$ the crucial step is to calculate

$$
\left.\frac{d}{d \lambda}\right|_{\lambda=0} \mathscr{L}_{W_{\lambda}} g_{\lambda}=-\left.\frac{d}{d \lambda}\right|_{\lambda=0} \delta^{*}\left[g_{\lambda}\right] \omega_{\lambda},
$$

where $\left(\omega_{\lambda}\right)_{i}:=\left(g_{\lambda}\right)_{i k} W_{\lambda}^{k}$ and $W_{\lambda}=-\tilde{g}^{-1} \delta\left[g_{\lambda}\right] G\left[g_{\lambda}\right](\tilde{g})$. In fact, the key point of DeTurck's trick is to collect the second-order covariant derivatives of $h$ in the above and realize that they cancel out the second line in (3-10). To see that we first collect terms involving the first-order covariant derivatives of $h$ in

$$
\left.\frac{d}{d \lambda}\right|_{\lambda=0}\left(\omega_{\lambda}\right)_{i}=\nabla^{k} h_{k i}-\frac{1}{2} \nabla_{i} h_{k}^{k}+\text { other terms. }
$$

Then we collect the second-order covariant derivatives of $h$ in

$$
\left.\frac{d}{d \lambda}\right|_{\lambda=0}\left(\left(\delta_{g_{\lambda}}\right)^{*} \omega_{\lambda}\right)_{i j}=-\nabla_{i} \nabla^{k} h_{k i}-\nabla_{j} \nabla^{k} h_{k j}+\nabla_{i} \nabla_{j} h_{k}^{k}+\text { other terms. }
$$

Therefore

$$
\left.\frac{d}{d \lambda}\right|_{\lambda=0} M\left(g_{\lambda}\right)=\frac{\partial}{\partial t} h-\Delta h+2 \mathscr{P}^{\prime} g+M_{1}^{i j k} \nabla_{i} h_{j k}+M_{2}^{i j} h_{i j},
$$

where $M_{1}$ depends only $g(t)$ and $\mathscr{P}(g)$ and $M_{2}$ depends on the curvature of $g(t)$ and $\mathscr{P}(g)$. To summarize, we have this:

Lemma 3.3. Suppose that $g(t), t \in[0, T]$, is a family of metrics such that the elliptic operator $(m-1) \Delta_{g(t)}+s_{0}$ is invertible for all $t \in[0, T]$. Then the linearization of the DeTurck conformal Ricci flow equations (3-2) at the metrics $g(t)$ in the directions of the symmetric 2-tensors $h(t)$ is

$$
D \mathcal{M}(g)(h)=\frac{\partial}{\partial t} h-\Delta h+2 \mathscr{P}^{\prime} g+M_{1} * \nabla h+M_{2} * h,
$$

where

$$
\mathscr{P}^{\prime}=\left((m-1) \Delta+s_{0}\right)^{-1}\left(P_{1} * \nabla^{2} h+P_{2} * \nabla h+P_{3} * h\right) .
$$

Here $P_{1}, P_{2}, P_{3}$ are tensors depending on curvature of $g(t)$ and up to the secondorder derivatives in spatial variables of $\mathscr{P}(g)$, and $M_{1}, M_{2}$ are tensors depending on the curvature of $g(t)$ and the function $\mathscr{P}(g)$. 
3.3. Short-time existence on closed manifolds. Let us first solve the conformal Ricci flow on a compact manifold $M^{m}$. There are many books that are good references for linear and nonlinear systems of parabolic equations. We will mostly use the book [Lunardi 1995, §5.1], in particular its Theorem 5.1.21, for existence and standard estimates. We adopt the definitions of parabolic Hölder spaces from [Lunardi 1995, pp. 175-177]. We use the same notations for parabolic Hölder spaces of functions and of tensor fields when there is no confusion. To define norms for tensor fields we may use the initial metric and local coordinate charts.

3.3.1. Preliminaries. Since we deal with systems of parabolic-elliptic equations, we need to consider elliptic estimates with a time parameter. There is an advantage to using only the supremum norm in the time variable, as indicated by the following lemma.

Lemma 3.4. Let $g(t), t \in[0, T]$, be a family of smooth Riemannian metrics on a compact manifold $M^{m}$. Suppose the operator $(m-1) \Delta_{g(t)}+s_{0}$ is invertible for $t \in[0, T]$. Then the equation

$$
(m-1) \Delta_{g(t)} p(t)+s_{0} p(t)=\gamma
$$

has a unique solution $p \in C^{0,2+\alpha}$ for each $\gamma \in C^{0, \alpha}$. Moreover, $p$ satisfies the estimate

$$
\|p\|_{C^{0,2+\alpha}} \leq C\|\gamma\|_{C^{0, \alpha}}
$$

for some constant $C$ independent of $\gamma$.

Proof. In the light of the standard Schauder estimates for elliptic PDEs, we only need to verify that $p(t)$ is continuous in the time variable, which is a consequence of the classical Bernstein estimates.

The following interpolatory inclusion will be useful in the proof of the short-time existence (cf. [Lunardi 1995, Lemma 5.1.1]).

Lemma 3.5. There is a constant $C$ independent of $T$ such that for any $t_{1}, t_{2} \in[0, T]$ we have

$$
\left\|h\left(t_{1}, \cdot\right)-h\left(t_{2}, \cdot\right)\right\|_{C^{k-2, \alpha}} \leq C \cdot\left|t_{1}-t_{2}\right| \cdot\|h\|_{C^{1, k+\alpha}}
$$

for all $h \in C^{1, k+\alpha}([0, T] \times M)$.

3.3.2. On linearized DeTurck conformal Ricci flow. We first solve the linearized DeTurck conformal Ricci flow

$$
\left\{\begin{aligned}
D \mathcal{M}(g)(h) & =\frac{\partial}{\partial t} h-\Delta h+2 \mathscr{P}^{\prime} g+M_{1} * \nabla h+M_{2} * h=\gamma, \\
h(0, \cdot) & =0
\end{aligned}\right.
$$

for appropriately given metrics $g(t)$ for each $\gamma \in C^{0, \alpha}$. 
Proposition 3.6. Suppose that $g(t), t \in[0, T]$, is a family of metrics such that the elliptic operator $(m-1) \Delta_{g(t)}+s_{0}$ is invertible for all $t \in[0, T]$. Then, for $\gamma \in C^{0, \alpha}$, the initial value problem for (3-17) has a unique solution $h \in C^{1,2+\alpha}$. Moreover,

$$
\|h\|_{C^{1,2+\alpha}([0, T] \times M)} \leq C\|\gamma\|_{C^{0, \alpha}([0, T] \times M)} .
$$

Proof. To use a contractive mapping-type argument we consider the Banach space

$$
E_{1}\left(\left[0, T^{*}\right]\right)=\left\{h \in C^{0,2+\alpha}: h(0, \cdot)=0\right\} .
$$

Given $\tilde{h} \in E_{1}\left(\left[0, T^{*}\right]\right)$, based on [Lunardi 1995, Theorem 5.1.21], we first solve a usual system of linear parabolic equations

$$
\left\{\begin{aligned}
\frac{\partial}{\partial t} h-\Delta h+M_{1} * \nabla h+M_{2} * h & =\tilde{\gamma}, \\
h(0, \cdot) & =0,
\end{aligned}\right.
$$

where $\tilde{\gamma}=\gamma-2 \mathscr{P}^{\prime}(\tilde{h}) g \in C^{0, \alpha}$ and $\mathscr{P}^{\prime}(\tilde{h})$ is defined by (3-11). We remark that it takes some work to extend Theorem 5.1.21 in [Lunardi 1995] to be applicable to our context, but there are no significant issues in doing so. Hence we may define a map

$$
\Psi: E_{1}\left(\left[0, T^{*}\right]\right) \rightarrow E_{1}\left(\left[0, T^{*}\right]\right), \quad \Psi(\tilde{h})=h .
$$

Note that if we set

$$
v=\Psi\left(\tilde{h}_{1}\right)-\Psi\left(\tilde{h}_{2}\right)
$$

then $v$ satisfies

$$
\left\{\begin{aligned}
\frac{\partial}{\partial t} v-\Delta v+M_{1} * \nabla v+M_{2} * v & =2\left(\mathscr{P}^{\prime}\left(\tilde{h}_{2}\right)-\mathscr{P}^{\prime}\left(\tilde{h}_{1}\right)\right) g \\
v(0, \cdot) & =0 .
\end{aligned}\right.
$$

Since

$$
\left\|\left(\mathscr{P}^{\prime}\left(\tilde{h}_{2}\right)-\mathscr{P}^{\prime}\left(\tilde{h}_{1}\right)\right) g\right\|_{C^{0,2+\alpha}} \leq C\left\|\tilde{h}_{1}-\tilde{h}_{2}\right\|_{C^{0,2+\alpha}}
$$

holds by (3-11) and Lemma 3.4, we obtain again from the estimates based on Theorem 5.1.21 in [Lunardi 1995] that

$$
\|v\|_{C^{1,4+\alpha}} \leq C\left\|\tilde{h}_{1}-\tilde{h}_{2}\right\|_{C^{0,2+\alpha}} .
$$

In the light of Lemma 3.5, we thus have

$$
\left\|v\left(t_{1}\right)-v\left(t_{2}\right)\right\|_{C^{2, \alpha}} \leq C \cdot\left|t_{1}-t_{2}\right| \cdot\left\|\tilde{h}_{1}-\tilde{h}_{2}\right\|_{C^{0,2+\alpha}} .
$$

In particular,

$$
\left\|\Psi\left(\tilde{h}_{1}\right)-\Psi\left(\tilde{h}_{2}\right)\right\|_{C^{0,2+\alpha}} \leq C T^{*}\left\|\tilde{h}_{1}-\tilde{h}_{2}\right\|_{C^{0,2+\alpha}} .
$$


To apply the contractive mapping theorem we observe that

$$
\|\Psi(\tilde{h})\|_{C^{0,2+\alpha}} \leq\|\Psi(0)\|_{C^{0,2+\alpha}}+C T^{*}\|\tilde{h}\|_{C^{0,2+\alpha}},
$$

where

$$
\|\Psi(0)\|_{C^{1,2+\alpha}} \leq C_{0}\|\gamma\|_{C^{0, \alpha}}
$$

for some constant $C_{0}$, from the estimates based on Theorem 5.1.21 in [Lunardi 1995]. Thus

$$
\Psi: B_{R}=\left\{h \in E_{1}\left(\left[0, T^{*}\right]\right):\|h\|_{C^{0,2+\alpha}} \leq R\right\} \rightarrow B_{R},
$$

for $R=2 C_{0}\|\gamma\|_{C^{0, \alpha}}$, is a contractive mapping when $T^{*}$ is appropriately small. Then, by the uniqueness of the solution of the linear parabolic equation (3-17), one may extend the solution of (3-17) to $[0, T]$ by steps in time of length $T^{*}$. The estimate (3-18) follows from the estimates based on Theorem 5.1.21 in [Lunardi 1995].

To summarize, we have established that

$$
D \mathcal{M}(g): C^{1,2+\alpha}([0, T] \times M) \cap\{h(0, \cdot)=0\} \rightarrow C^{0, \alpha}([0, T] \times M)
$$

is an isomorphism, provided that $g(t)$ satisfies the assumptions in Proposition 3.6.

3.3.3. Implicit function theorem argument. Next we solve the DeTurck conformal Ricci flow and then the conformal Ricci flow. Our approach is to use an implicit function theorem. Let us start with the following general implicit function theorem.

Lemma 3.7. Let $X$ and $Y$ be Banach spaces and let

$$
\mathscr{H}: X \rightarrow Y
$$

be a $C^{1}$ map. Suppose that for a point $x_{0} \in X$ there are positive numbers $\delta$ and $C$ such that

$$
\begin{array}{cc}
\left\|(D \mathscr{H}(x))^{-1}\right\| \leq C \quad & \text { for all } x \in B_{\delta}\left(x_{0}\right), \\
\left\|D \mathscr{H}\left(x_{1}\right)-D \mathscr{H}\left(x_{2}\right)\right\| \leq \frac{1}{2 C} & \text { for all } x_{1}, x_{2} \in B_{\delta}\left(x_{0}\right) .
\end{array}
$$

Then, if

$$
\left\|\mathscr{H}\left(x_{0}\right)\right\| \leq \frac{\delta}{2 C}
$$

there is $x \in B_{\delta}\left(x_{0}\right)$ such that

$$
\mathscr{H}(x)=0 .
$$

To apply the above implicit function theorem to the map

$$
\mathcal{M}: C^{1,2+\alpha}([0, T] \times M) \cap\left\{g(0)=g_{0}\right\} \rightarrow C^{0, \alpha}([0, T] \times M)
$$


to solve the DeTurck conformal Ricci flow we need to show that $M$ is continuously differentiable. In fact we have the following lemma.

Lemma 3.8. Let $M^{m}$ be a compact manifold and let $g(t) \in C^{1,2+\alpha}([0, T] \times M)$ be a family of metrics such that the elliptic operator $(m-1) \Delta_{g(t)}+s_{0}$ is invertible for all $t \in[0, T]$. Then there is a $\delta_{0}>0$ such that

$$
\left\|D M\left(g_{1}\right)-D M\left(g_{2}\right)\right\|_{L\left(C^{1,2+\alpha}, C^{0, \alpha}\right)} \leq C\left\|g_{1}-g_{2}\right\|_{C^{1,2+\alpha}}
$$

for $\left\|g_{i}-g\right\|_{C^{1,2+\alpha}} \leq \delta_{0}$ in $C^{1,2+\alpha}([0, T] \times M)$ and $i=1,2$.

Proof. We calculate, for any $h \in C^{1,2+\alpha} \bigcap\{h(0, \cdot)=0\}$, that

$$
\begin{aligned}
\left(D \mathcal{M}\left(g_{1}\right)-D \mathcal{M}\left(g_{2}\right)\right) h= & \left(\Delta_{g_{2}}-\Delta_{g_{1}}\right) h+2 \mathscr{P}^{\prime}\left[g_{1}\right]\left(g_{1}-g_{2}\right) \\
& +M_{1}\left[g_{1}\right] *\left(\nabla_{g_{1}} h-\nabla_{g_{2}} h\right)+\left(M_{1}\left[g_{1}\right]-M_{1}\left[g_{2}\right]\right) * \nabla_{g_{2}} h \\
& +\left(M_{2}\left[g_{1}\right]-M_{2}\left[g_{2}\right]\right) * h+2\left(\mathscr{P}^{\prime}\left[g_{1}\right]-\mathscr{P}^{\prime}\left[g_{2}\right]\right) g_{2} .
\end{aligned}
$$

It is easily seen that

$$
\begin{aligned}
\left\|M_{1}\left[g_{1}\right] *\left(\nabla_{g_{1}} h-\nabla_{g_{2}} h\right)+\left(M_{1}\left[g_{1}\right]-M_{1}\left[g_{2}\right]\right) * \nabla_{g_{2}} h\right\|_{C^{0, \alpha}} & \\
& \leq C\left\|g_{1}-g_{2}\right\|_{C^{1,2+\alpha}}\|h\|_{C^{1,2+\alpha}}, \\
\left\|\left(M_{2}\left[g_{1}\right]-M_{2}\left[g_{2}\right]\right) h\right\|_{C^{0, \alpha}} & \leq C\left\|g_{1}-g_{2}\right\|_{C^{1,2+\alpha}}\|h\|_{C^{1,2+\alpha}}, \\
\left\|\Delta_{g_{1}} h-\Delta_{g_{2}} h\right\|_{C^{0, \alpha}} & \leq C\left\|g_{1}-g_{2}\right\|_{C^{1,2+\alpha}}\|h\|_{C^{1,2+\alpha}} .
\end{aligned}
$$

It is also easy to see that

$$
\left\|\mathscr{P}^{\prime}\left[g_{1}\right]\left(g_{1}-g_{2}\right)\right\|_{C^{0, \alpha}} \leq C\left\|g_{1}-g_{2}\right\|_{C^{1,2+\alpha}}\|h\|_{C^{1,2+\alpha}}
$$

under the assumption that $\left\|g_{i}-g\right\|_{C^{1,2+\alpha}} \leq \delta_{0}, i=1,2$, from the definition of $\mathscr{P}^{\prime}$ in (3-11).

For the last remaining term we write

$$
\begin{aligned}
\left((m-1) \Delta+s_{0}\right)\left(\mathscr{P}^{\prime}\left[g_{2}\right]-\mathscr{P}^{\prime}\left[g_{1}\right]\right)=(m-1)\left(\Delta_{g_{1}}-\Delta_{g_{2}}\right) \mathscr{P}^{\prime}\left[g_{1}\right] \\
+\left(P_{1}\left[g_{2}\right]-P_{1}\left[g_{1}\right]\right) * \nabla_{g_{2}}^{2} h+P_{1}\left[g_{1}\right] *\left(\nabla_{g_{2}}^{2}-\nabla_{g_{1}}^{2}\right) h \\
+\left(P_{2}\left[g_{2}\right]-P_{2}\left[g_{1}\right]\right) * \nabla_{g_{2}} h+P_{2}\left[g_{1}\right] *\left(\nabla_{g_{2}}-\nabla_{g_{1}}\right) h \\
+\left(P_{3}\left[g_{2}\right]-P_{3}\left[g_{1}\right]\right) * h
\end{aligned}
$$

and apply Lemma 3.4. Then

$$
\left\|\mathscr{P}^{\prime}\left[g_{1}\right]-\mathscr{P}^{\prime}\left[g_{2}\right]\right\|_{C^{0, \alpha}} \leq C\left\|g_{1}-g_{2}\right\|_{C^{1,2+\alpha}}\|h\|_{C^{1,2+\alpha}},
$$

which implies that

$$
\left\|\left(\mathscr{P}^{\prime}\left[g_{1}\right]-\mathscr{P}^{\prime}\left[g_{2}\right]\right) g\right\|_{C^{0, \alpha}} \leq C\left\|g_{1}-g_{2}\right\|_{C^{1,2+\alpha}}\|h\|_{C^{1,2+\alpha}} .
$$

Thus the proof is complete. 
To apply Lemma 3.7, we consider the initial approximate solution

$$
\bar{g}(t)=g_{0}+t \mathscr{F}\left(g_{0}\right),
$$

where $\mathscr{F}$ was introduced in (3-9). We then calculate that

$$
\begin{aligned}
\mathcal{M}(\bar{g}) & =-\mathscr{F}\left(g_{0}+t \mathscr{F}\left(g_{0}\right)\right)+\mathscr{F}\left(g_{0}\right) \\
& =-t \int_{0}^{1} D \mathscr{F}\left(g_{0}+\theta t \mathscr{F}\left(g_{0}\right)\right) d \theta \cdot \mathscr{F}\left(g_{0}\right) .
\end{aligned}
$$

Now we are ready to state and prove the short-time existence theorem for the conformal Ricci flow (a precise form of Theorem 1.1).

Theorem 3.9. Let $M^{m}$ be a compact manifold with no boundary. Suppose that $g_{0} \in C^{4, \alpha}$ is a Riemannian metric on $M$ such that the scalar curvature $s_{g_{0}}=s_{0}$ is constant and that the elliptic operator $(m-1) \Delta_{g_{0}}+s_{0}$ is invertible. Then there exists a small positive number $T$ such that the conformal Ricci flow $g(t)$ exists in $C^{1,2+\alpha}$ from the initial metric $g_{0}$ for $t \in[0, T]$.

Proof. First we notice that Proposition 3.6 holds for the family of metrics $\bar{g}(t)=$ $g_{0}+t \mathscr{F}\left(g_{0}\right)$ in $C^{1,2+\alpha}$, for some appropriately small $T$ such that the elliptic operator $(m-1) \Delta_{\bar{g}}+s_{0}$ is invertible for all $t \in[0, T]$. Therefore there is a constant $C$ and a small number $\delta_{0}$ such that

$$
\left\|(D M(g))^{-1}\right\| \leq C \quad \text { and } \quad\left\|D M\left(g_{1}\right)-D M\left(g_{2}\right)\right\| \leq \frac{1}{2 C}
$$

for all $g, g_{1}, g_{2}, \in B\left(\delta_{0}\right)$, where $B\left(\delta_{0}\right)=\left\{g \in C^{1,2+\alpha}:\|g-\bar{g}\|_{C^{1,2+\alpha}} \leq \delta_{0}\right\}$, according to Lemma 3.8. Next, after choosing an even smaller $T$ if necessary, we observe from (3-21) that

$$
\|M(\bar{g})\|_{C^{0, \alpha}} \leq \frac{\delta_{0}}{2 C} .
$$

Hence Lemma 3.7 implies that DeTurck conformal Ricci flow $\hat{g}(t)$ exists in $C^{1,2+\alpha}$ with the initial metric $g_{0}$. Therefore, applying Lemma 3.1, we obtain the short-time existence for conformal Ricci flow from the initial metric $g_{0}$, since (3-7) is always solvable for short time.

3.4. Short-time existence on asymptotically flat manifolds. In this subsection we establish the short-time existence of the conformal Ricci flow on asymptotically flat manifolds. The idea of the proof is the same as of the proof in last subsection. We remark here that the short-time existence of the Ricci flow on asymptotically flat manifolds has been established independently in [Dai and Ma 2007; Oliynyk and Woolgar 2007]. The approach in [Dai and Ma 2007] is to use the short-time existence result in [Shi 1989] and the maximum principle to show that the Ricci flow in fact remains asymptotically flat when starting from an asymptotically flat 
metric, while the approach in [Oliynyk and Woolgar 2007] is to establish short-time existence of the Ricci flow based on weighted function spaces. Our approach is similar to the one in [Oliynyk and Woolgar 2007], since neither short-time existence on noncompact manifolds nor a maximum principle are available for the conformal Ricci flow.

3.4.1. Analysis on asymptotically flat manifolds. We first briefly introduce asymptotically flat manifolds according to [Lee and Parker 1987] and then construct appropriate parabolic Hölder spaces on them.

Definition 3.10 [Lee and Parker 1987, Definition 6.3]. A Riemannian manifold $M^{m}$ with a $C^{2}$-metric $g$ is called asymptotically flat of order $\tau>0$ if there exists a decomposition $M=M_{0} \cup M_{\infty}$ (with $M_{0}$ compact) and a diffeomorphism $\Psi$ : $M_{\infty} \rightarrow \mathbb{R}^{n} \backslash B_{R}(\overrightarrow{0})$ for some $R>0$, satisfying

$$
g(z)=g_{e}(z)+O\left(\rho^{-\tau}\right), \quad \partial_{k} g(z)=O\left(\rho^{-\tau-1}\right), \quad \partial_{k} \partial_{l} g(z)=O\left(\rho^{-\tau-2}\right),
$$

where $g_{e}$ is the standard Euclidean metric and $\rho=\rho(z)=|z| \rightarrow \infty$ in the coordinates $z=\left(z^{1}, \cdots, z^{m}\right)$ induced on $M_{\infty}$ by the diffeomorphism $\Psi$.

We give the definition of weighted Hölder spaces $C_{\beta}^{k, \alpha}$ from [Lee and Parker 1987, p. 75]. Again we will use the same notations for weighted Hölder spaces of functions and of tensor fields if there is no confusion. We use local coordinate charts and a given metric whenever it is necessary for the definition of Hölder spaces of tensor fields on asymptotically flat manifolds.

Fix a number $T>0$. Analogous to [Lunardi 1995, pp. 175-177], we define a parabolic weighted Hölder space

$$
C_{\beta}^{0, k+\alpha}:=\left\{h \in C(M \times[0, T]): h(t) \in C_{\beta}^{k, \alpha} \text { and } \max _{t \in[0, T]}\|h(t)\|_{C_{\beta}^{k, \alpha}}<\infty\right\}
$$

with the norm

$$
\|h\|_{C_{\beta}^{0, k+\alpha}}:=\max _{t \in[0, T]}\|h(t)\|_{C_{\beta}^{k, \alpha}} .
$$

Similarly we define the space

$$
C_{\beta}^{1, k+\alpha}:=\left\{h \in C_{\beta}^{0, k+\alpha} \text { and } \partial_{t} h \in C_{\beta-2}^{0, k-2+\alpha}\right\}
$$

with the norm

$$
\|h\|_{C_{\beta}^{1, k+\alpha}}:=\max _{t \in[0, T]}\|h(t)\|_{C_{\beta}^{k, \alpha}}+\max _{t \in[0, T]}\left\|\partial_{t} h(t)\right\|_{C_{\beta-2}^{k-2, \alpha}} .
$$

We now recall the elliptic theory for weighted Hölder spaces, for example, from [Lee and Parker 1987, Theorem 9.2] in our context. 
Lemma 3.11. Let $\left(M^{m}, g(t)\right)$, for $t \in[0, T]$, be a family of asymptotically flat manifolds with $g(t)-g_{e} \in C_{-\tau}^{0,2+\alpha}$ for $\tau>0$. Then

$$
\Delta_{g(t)}: C_{\beta}^{0,2+\alpha} \rightarrow C_{\beta-2}^{0, \alpha}
$$

is an isomorphism for $\beta \in(2-m, 0)$, that is, there is $C$ such that

$$
\|u\|_{C_{\beta}^{0,2+\alpha}} \leq C\left\|\Delta_{g(t)} u\right\|_{C_{\beta-2}^{0, \alpha}} .
$$

Analogous to Lemma 3.5 we have a simple interpolatory inclusion.

Lemma 3.12. There is a constant $C$ independent of $T$ such that for any $t_{1}, t_{2} \in$ $[0, T]$, we have

$$
\left\|h\left(\cdot, t_{1}\right)-h\left(\cdot, t_{2}\right)\right\|_{C_{\beta-2}^{k-2, \alpha}} \leq C \cdot\left|t_{1}-t_{2}\right| \cdot\|h\|_{C_{\beta}^{1, k+\alpha}}
$$

for all $h \in C_{\beta}^{1, k+\alpha}(M \times[0, T])$.

3.4.2. Short-time existence on asymptotically flat manifolds. Here we assume that the initial metric $g_{0}$ on $M^{m}$ is asymptotically flat and scalar flat. Thanks to [Schoen and Yau 1979, Lemma 3.3 and Corollary 3.1], we know that one can always conformally deform an asymptotically flat metric with nonnegative scalar curvature into a scalar flat asymptotically flat metric. We will use the strategy of Section 3.3 to prove the short-time existence of conformal Ricci flow on asymptotically flat manifolds.

First with changes of notations we are able to prove the isomorphism analogous to Proposition 3.6. An extension of [Lunardi 1995, Theorem 5.1.21] to the weighted parabolic Hölder spaces on asymptotically flat manifolds may be proven by the standard argument through interior estimates and scaling invariance of the interior estimates (cf. [Oliynyk and Woolgar 2007; Bartnik 1986; Lee and Parker 1987]). The key is to realize that one may move in and out the weight for local estimates.

Proposition 3.13. Suppose that $g(t), t \in\left[0, T_{0}\right]$, is a family of asymptotically flat metrics with $g(t)-g_{e} \in C_{-\tau}^{0,2+\alpha}$ for $\tau \in(0, m-2)$. Then there is a $T_{*} \in\left(0, T_{0}\right]$ such that, for any $T \leq T_{*}$ and $\gamma \in C_{-\tau-2}^{0, \alpha}$, the initial value problem for (3-17) has a unique solution $h \in C_{-\tau}^{1,2+\alpha}$. Moreover,

$$
\|h\|_{C_{-\tau}^{1,2+\alpha}(M \times[0, T])} \leq C\|\gamma\|_{C_{-\tau-2}^{0, \alpha}(M \times[0, T])} .
$$

This is to say that, for $\tau \in(0, m-2)$,

$$
\operatorname{DM}(g): C_{-\tau}^{1,2+\alpha}(M \times[0, T]) \cap\{h(0, \cdot)=0\} \rightarrow C_{-\tau-2}^{0, \alpha}(M \times[0, T])
$$

is an isomorphism, provided that $g(t)$ and $T$ satisfy the assumptions in the above Proposition 3.13. The restriction on the order $\tau$ of weight is solely used in solving elliptic equations on weighted spaces in Lemma 3.11. 
To obtain a short-time existence of the DeTurck conformal Ricci flow we again apply an implicit function theorem (Lemma 3.7) to the map

$\mathcal{M}:\left\{g(t): g(t)-g_{e} \in C_{-\tau}^{1,2+\alpha}(M \times[0, T])\right.$ and $\left.g(0)=g_{0}\right\} \rightarrow C_{-\tau-2}^{0, \alpha}(M \times[0, T])$

for any $\tau \in(0, m-2)$ and $T$ given from Proposition 3.13. Finally, we arrive at the short-time existence of the conformal Ricci flow.

Theorem 3.14. Let $\left(M^{m}, g_{0}\right)$ be scalar flat and asymptotically flat with $g_{0}-g_{e} \in$ $C_{-\tau}^{4, \alpha}$ and $\tau \in(0, m-2)$. Then there exists a small positive number $T$ such that the conformal Ricci flow $g(t)$ from the initial metric $g_{0}$ exists for $t \in[0, T]$ and $g(t)-g_{e} \in C_{-\tau}^{1,2+\alpha}(M \times[0, T])$.

Proof. As in Section 3.3.3 we first verify that

$$
\left\|\operatorname{DM}\left(g_{1}\right)-\operatorname{DM}\left(g_{2}\right)\right\|_{L\left(C_{-\tau}^{1,2+\alpha}, C_{-\tau-2}^{0, \alpha}\right)} \leq C\left\|g_{1}-g_{2}\right\|_{C_{-\tau}^{1,2+\alpha}} .
$$

The proof goes like the one for Lemma 3.8 with only changes of notation. We then construct

$$
\bar{g}(t)=g_{0}+t \mathscr{F}\left(g_{0}\right) \in g_{e}+C_{-\tau}^{1,2+\alpha}
$$

as in Section 3.3.3 for $g_{0}-g_{e} \in C_{-\tau}^{4, \alpha}$. Another issue one needs to take care of is solving (3-7) to construct the conformal Ricci flow from the DeTurck conformal Ricci flow. But, since $W \in C_{-\tau-1}^{0,1+\alpha}$, it is easy to solve (3-7) on the whole manifold $M$ for some short time. The rest of the proof goes like the one in Section 3.3.3 for Theorem 3.9 with little changes except in notation. Notice that the equivalence between (1-1) and (1-2) holds because of the uniqueness of the bounded solution of a linear parabolic equation on an asymptotically flat manifold.

\section{ADM mass under conformal Ricci flow}

Asymptotically flat manifolds are used in general relativity to describe isolated gravitational systems. The fundamental geometric invariant of an asymptotically flat manifold is called the mass of the gravitational system. The so-called ADM mass of an asymptotically flat manifold was first defined in [Arnowitt et al. 1960].

In general relativity the world is modeled by a 4-dimensional spacetime $X^{4}$ with a Lorentzian metric $g$. The physical law that describes the gravity induced by matter in the spacetime is the famous Einstein equation

$$
\operatorname{Ric}_{g}-\frac{1}{2} s_{g} g=T,
$$

where $T$ is the energy-momentum-stress tensor that is supposed to reflect the nature and state of matter in the spacetime. A time slice of a space-time that represents an isolated gravitational system is an asymptotically flat 3-manifold $M^{3}$. 
One of the most important solutions of Einstein equations is the Schwarzschild spacetime, which represents the gravitational system of a static point particle of mass $\boldsymbol{m}$ and whose time slice is an asymptotically flat metric

$$
g_{\mathrm{Sch}}=g_{e}+\frac{\boldsymbol{m}}{\rho} g_{e}+O\left(\rho^{-2}\right)
$$

on the punctured $\mathbb{R}^{3}$. The crucial test to validate the notion of mass in relativity is whether its predictions reduce to those of Newtonian gravity under the circumstances where Newtonian theory is known to be valid; when gravity is weak, motions are much slower than the speed of the light, and material stresses are much smaller than the mass-energy density (cf. [Wald 1984, 4.4]).

We now follow [Lee and Parker 1987, Definition 8.2] to introduce ADM mass for asymptotically flat manifolds.

Definition 4.1. Given an asymptotically flat Riemannian manifold $\left(M^{m}, g\right)$ with asymptotic coordinates $z$, we define the ADM mass by (if the limit exists)

$$
\boldsymbol{m}(g)=\lim _{R \rightarrow \infty} \omega_{m-1}^{-1} \int_{\mathbb{S}_{R}}\left(\partial_{i} g_{i j}-\partial_{j} g_{i i}\right) n^{j} d \sigma,
$$

where $\omega_{m-1}$ is the volume of the unit sphere $\mathbb{S}^{m-1}, \vec{n}=\left(n^{1}, \ldots, n^{m}\right)$ is the outward unit normal vector of the sphere $\mathbb{S}_{R}=\left\{z \in \mathbb{R}^{m},|z|=R\right\}$ and $d \sigma$ is the area element of $\mathbb{S}_{R}$.

Recall from [Lee and Parker 1987] that

$$
\mathcal{M}_{\tau}:=\left\{g=g_{e}+h: h \in C_{-\tau}^{1, \alpha} \text { and } \partial_{j} \partial_{i} h_{i j}-\partial_{j} \partial_{j} h_{i i} \in L^{1}\left(M, d \operatorname{vol}_{g_{e}}\right)\right\} .
$$

After Definition 4.1 one wonders if the ADM mass is indeed a geometric invariant for the asymptotically flat metric. This was confirmed by the following result:

Lemma 4.2 [Arnowitt et al. 1960; Bartnik 1986]. Suppose that $g$ is an asymptotically flat metric in $\mathcal{M}_{\tau}$ on $M^{m}$ for $\tau>\frac{m-2}{2}$. Then the ADM mass $\boldsymbol{m}(g)$ is independent of the choice of asymptotic coordinates at infinity.

Another important fact about the ADM mass is the following, observed in [Lee and Parker 1987, (8.11); see also Lemma 9.4].

Lemma 4.3. Let $g(t)$ be a smooth family of asymptotically flat metrics in $\mu_{\tau}$ on $M^{m}$, for $\tau>\frac{m-2}{2}$. Then the mass $\boldsymbol{m}(g(t))$ is differentiable and

$$
\frac{d}{d t}\left(-\int_{M} s_{g(t)} d \operatorname{vol}_{g(t)}+\omega_{m-1} \boldsymbol{m}(g(t))\right)=\int_{M} G[g(t)] \cdot \varphi(t) d \operatorname{vol}_{g(t)},
$$

where $G[g(t)]=\operatorname{Ric}_{g(t)}-\frac{1}{2} s_{g(t)} g(t)$ is the Einstein tensor and $\varphi(t)=\partial_{t} g(t)$.

Theorem 3.14 and Lemma 4.3 now entail the following theorem. 
Theorem 4.4. Let $g_{0}$ be a scalar flat and asymptotically flat metric on $M^{m}$ such that $g_{0}-g_{e} \in C_{-\tau}^{4, \alpha}$ for $\tau \in\left(\frac{m-2}{2}, m-2\right)$. Then the conformal Ricci flow $g(t)$ starting with $g(0)=g_{0}$ exists for some short time and

$$
g(t) \in \mathcal{M}_{\tau} \quad \text { and } \quad g(t)-g_{e} \in C_{-\tau}^{1,2+\alpha} .
$$

Moreover,

$$
\frac{d}{d t} \boldsymbol{m}(g(t))=-2 \int_{M}\left|\operatorname{Ric}_{g(t)}\right|^{2} d \operatorname{vol}_{g(t)} .
$$

In particular, the ADM mass is strictly decreasing under conformal Ricci flow, except when $g_{0}$ is the Euclidean metric.

Proof. To verify that the conformal Ricci flow $g(t)$ stays in $\mu_{\tau}$ we only need to verify that

$$
\partial_{j} \partial_{i} g_{i j}(t)-\partial_{j} \partial_{j} g_{i i}(t) \in L^{1}\left(M, d \operatorname{vol}_{g_{e}}\right) .
$$

Recall that [Lee and Parker 1987, (9.2)]

$$
s=\partial_{j} \partial_{i} g_{i j}-\partial_{j} \partial_{j} g_{i i}+O\left(\rho^{-2 \tau-2}\right),
$$

which implies that

$$
\partial_{j} \partial_{i} g_{i j}-\partial_{j} \partial_{j} g_{i i}=O\left(\rho^{-2 \tau-2}\right) \in L^{1}\left(M, d \operatorname{vol}_{g_{e}}\right)
$$

for $\tau \in\left(\frac{m-2}{2}, m-2\right)$. It is easily seen that the ADM mass is strictly decreasing except when $g_{0}$ is Ricci flat. Then, using [Bando et al. 1989, Theorem 1.5] and [Lee and Parker 1987, Proposition 10.2], one concludes that $g_{0}$ is the standard Euclidean metric. Therefore the proof is complete.

A quick application of the above Theorem 4.4 is a simple and direct proof of the rigidity part of Schoen and Yau's positive mass theorem.

Corollary 4.5 [Schoen and Yau 1979]. Suppose that $\left(M^{m}, g\right)$ is asymptotically flat manifold with nonnegative scalar curvature and that $g-g_{e} \in C_{-\tau}^{4, \alpha}$ for $\tau>\frac{m-2}{2}$. If the ADM mass $\boldsymbol{m}(\mathrm{g})$ is zero, then $(M, g)$ is isometric to the standard Euclidean space $\mathbb{R}^{m}$.

Proof. First we know that $g$ has to be scalar flat. Otherwise one can conformally deform the metric to a scalar flat one and decrease the ADM mass to be negative, which is impossible due to the first part of the positive mass theorem of Schoen and Yau. Next we invoke Theorem 4.4 and come to the same contradiction if $g$ is not flat. 


\section{Acknowledgment}

We would like to mention that Lars Andersson and Dan Lee have communicated to us that they are also aware of some version of Theorem 1.4. We are grateful that Dan Lee brought a related paper [Haslhofer 2011] to our attention.

Part of this work was done while the authors were visiting Beijing International Center for Mathematical Research and East China Normal University. The authors are grateful to them for the hospitality.

\section{References}

[Anderson 2005] M. T. Anderson, "On uniqueness and differentiability in the space of Yamabe metrics", Commun. Contemp. Math. 7:3 (2005), 299-310. MR 2006d:53031 Zbl 1082.58013

[Arnowitt et al. 1960] R. Arnowitt, S. Deser, and C. W. Misner, "Canonical variables for general relativity”, Phys. Rev. (2) 117 (1960), 1595-1602. MR 22 \#4505b Zbl 0091.21203

[Bando et al. 1989] S. Bando, A. Kasue, and H. Nakajima, "On a construction of coordinates at infinity on manifolds with fast curvature decay and maximal volume growth", Invent. Math. 97:2 (1989), 313-349. MR 90c:53098 Zbl 0682.53045

[Bartnik 1986] R. Bartnik, "The mass of an asymptotically flat manifold”, Comm. Pure Appl. Math. 39:5 (1986), 661-693. MR 88b:58144 Zbl 0598.53045

[Besse 1987] A. L. Besse, Einstein manifolds, Ergebnisse der Mathematik und ihrer Grenzgebiete (3) 10, Springer, Berlin, 1987. MR 88f:53087 Zbl 0613.53001

[Chang and Lu 2007] S.-C. Chang and P. Lu, "Evolution of Yamabe constant under Ricci flow", Ann. Global Anal. Geom. 31:2 (2007), 147-153. MR 2008e:53121 Zbl 1112.53048

[Chow et al. 2006] B. Chow, P. Lu, and L. Ni, Hamilton's Ricci flow, Grad. Studies Math. 77, Amer. Math. Soc., Providence, RI, 2006. MR 2008a:53068 Zbl 1118.53001

[Dai and Ma 2007] X. Dai and L. Ma, "Mass under the Ricci flow", Comm. Math. Phys. 274:1 (2007), 65-80. MR 2008h:53121 Zbl 1127.53056

[DeTurck 1983] D. M. DeTurck, "Deforming metrics in the direction of their Ricci tensors", $J$. Differential Geom. 18:1 (1983), 157-162. MR 85j:53050 Zbl 0517.53044

[DeTurck 2003] D. M. DeTurck, "Deforming metrics in the direction of their Ricci tensors: Improved version", pp. 163-165 in Collected papers on Ricci flow, edited by H.-D. Cao et al., Series in Geometry and Topology 37, International Press, Somerville, MA, 2003.

[Fischer 2004] A. E. Fischer, "An introduction to conformal Ricci flow", Classical Quantum Gravity 21:3 (2004), 171-218. MR 2005b:53109 Zbl 1050.53029 arXiv math/0312519

[Hamilton 1995] R. S. Hamilton, "The formation of singularities in the Ricci flow", pp. 7-136 in Proceedings of the Conference on Geometry and Topology (Cambridge, MA, 1993), edited by C.-C. Hsiung and S.-T. Yau, Surveys in Differential Geometry 2, International Press, Cambridge, MA, 1995. MR 97e:53075 Zbl 0867.53030

[Haslhofer 2011] R. Haslhofer, “A mass-decreasing flow in dimension three”, preprint, 2011. arXiv 1107.3220

[Koiso 1979] N. Koiso, "A decomposition of the space $M$ of Riemannian metrics on a manifold", Osaka J. Math. 16:2 (1979), 423-429. MR 80g:53034 Zbl 0416.58007

[Lee and Parker 1987] J. M. Lee and T. H. Parker, "The Yamabe problem”, Bull. Amer. Math. Soc. (N.S.) 17:1 (1987), 37-91. MR 88f:53001 Zbl 0633.53062 
[Lunardi 1995] A. Lunardi, Analytic semigroups and optimal regularity in parabolic problems, Progress Nonlinear Diff. Eq. and Appl. 16, Birkhäuser, Basel, 1995. MR 96e:47039 Zbl 0816.35001

[Oliynyk and Woolgar 2007] T. A. Oliynyk and E. Woolgar, "Rotationally symmetric Ricci flow on asymptotically flat manifolds", Comm. Anal. Geom. 15:3 (2007), 535-568. MR 2009b:53110 Zbl 1138.53057

[Schoen and Yau 1979] R. Schoen and S. T. Yau, "On the proof of the positive mass conjecture in general relativity”, Comm. Math. Phys. 65:1 (1979), 45-76. MR 80j:83024 Zbl 0405.53045

[Shi 1989] W.-X. Shi, "Ricci deformation of the metric on complete noncompact Riemannian manifolds", J. Differential Geom. 30:2 (1989), 303-394. MR 90f:53080 Zbl 0686.53037

[Wald 1984] R. M. Wald, General relativity, University of Chicago Press, Chicago, IL, 1984. MR 86a:83001 Zbl 0549.53001

[Wang and Zheng 2011] E.-M. Wang and Y. Zheng, "Regularity of the first eigenvalue of the $p$ Laplacian and Yamabe invariant along geometric flows", Pacific J. Math. 254:1 (2011), 239-255. MR 2900670 Zbl 06029190

Received April 20, 2012.

PENG LU

DEPARTMENT OF MATHEMATICS

UNIVERSITY OF OREGON

304 DEADY HALL

EUGENE, OR 97403

UNITED STATES

penglu@uoregon.edu

http://pages.uoregon.edu/penglu/

JIE QING

DEPARTMENT OF MATHEMATICS

UniVERSITY OF CALIFORNIA, SANTA CRUZ

MCHENRY 4178

SANTA CRUZ, CA 95064

UNITED STATES

qing@ucsc.edu

http://www.math.ucsc.edu/faculty/qing.html

YU ZHENG

DEPARTMENT OF MATHEMATICS

SHANGHAI KEY LABORATORY OF PMMP

EAST CHINA NORMAL UNIVERSITY

DONGCHUAN RD 500

SHANGHAI 200241

CHINA

zhyu@math.ecnu.edu.cn 


\title{
PACIFIC JOURNAL OF MATHEMATICS
}

\author{
msp.org/pjm
}

Founded in 1951 by E. F. Beckenbach (1906-1982) and F. Wolf (1904-1989)

\section{EDITORS}

Don Blasius (Managing Editor)

Department of Mathematics

University of California

Los Angeles, CA 90095-1555

blasius@math.ucla.edu

\author{
Paul Balmer \\ Department of Mathematics \\ University of California \\ Los Angeles, CA 90095-1555 \\ balmer@math.ucla.edu \\ Robert Finn \\ Department of Mathematics \\ Stanford University \\ Stanford, CA 94305-2125 \\ finn@math.stanford.edu \\ Sorin Popa \\ Department of Mathematics \\ University of California \\ Los Angeles, CA 90095-1555 \\ popa@math.ucla.edu
}

\author{
Vyjayanthi Chari \\ Department of Mathematics \\ University of California \\ Riverside, CA 92521-0135 \\ chari@math.ucr.edu \\ Kefeng Liu \\ Department of Mathematics \\ University of California \\ Los Angeles, CA 90095-1555 \\ liu@math.ucla.edu \\ Jie Qing \\ Department of Mathematics \\ University of California \\ Santa Cruz, CA 95064 \\ qing@ cats.ucsc.edu
}

\section{PRODUCTION}

Silvio Levy, Scientific Editor, production@msp.org

\section{SUPPORTING INSTITUTIONS}

ACADEMIA SINICA, TAIPEI

CALIFORNIA INST. OF TECHNOLOGY

INST. DE MATEMÁTICA PURA E APLICADA

KEIO UNIVERSITY

MATH. SCIENCES RESEARCH INSTITUTE

NEW MEXICO STATE UNIV.

OREGON STATE UNIV.

\author{
STANFORD UNIVERSITY \\ UNIV. OF BRITISH COLUMBIA \\ UNIV. OF CALIFORNIA, BERKELEY \\ UNIV. OF CALIFORNIA, DAVIS \\ UNIV. OF CALIFORNIA, LOS ANGELES \\ UNIV. OF CALIFORNIA, RIVERSIDE \\ UNIV. OF CALIFORNIA, SAN DIEGO \\ UNIV. OF CALIF., SANTA BARBARA
}

\author{
Daryl Cooper \\ Department of Mathematics \\ University of California \\ Santa Barbara, CA 93106-3080 \\ cooper@math.ucsb.edu \\ Jiang-Hua Lu \\ Department of Mathematics \\ The University of Hong Kong \\ Pokfulam Rd., Hong Kong \\ jhlu@maths.hku.hk \\ Paul Yang \\ Department of Mathematics \\ Princeton University \\ Princeton NJ 08544-1000 \\ yang@math.princeton.edu
}

These supporting institutions contribute to the cost of publication of this Journal, but they are not owners or publishers and have no responsibility for its contents or policies.

See inside back cover or msp.org/pjm for submission instructions.

The subscription price for 2014 is US $\$ 410 /$ year for the electronic version, and \$535/year for print and electronic.

Subscriptions, requests for back issues and changes of subscribers address should be sent to Pacific Journal of Mathematics, P.O. Box 4163, Berkeley, CA 94704-0163, U.S.A. The Pacific Journal of Mathematics is indexed by Mathematical Reviews, Zentralblatt MATH, PASCAL CNRS Index, Referativnyi Zhurnal, Current Mathematical Publications and Web of Knowledge (Science Citation Index).

The Pacific Journal of Mathematics (ISSN 0030-8730) at the University of California, c/o Department of Mathematics, 798 Evans Hall \#3840, Berkeley, CA 94720-3840, is published twelve times a year. Periodical rate postage paid at Berkeley, CA 94704, and additional mailing offices. POSTMASTER: send address changes to Pacific Journal of Mathematics, P.O. Box 4163, Berkeley, CA 94704-0163.

PJM peer review and production are managed by EditFLOW ${ }^{\circledR}$ from Mathematical Sciences Publishers.

\section{PUBLISHED BY}

\section{mathematical sciences publishers \\ nonprofit scientific publishing}

http://msp.org/

(C) 2014 Mathematical Sciences Publishers 


\title{
PACIFIC JOURNAL OF MATHEMATICS
}

\author{
Volume $268 \quad$ No. $2 \quad$ April 2014
}

In memoriam: Jonathan Rogawski

DON BLASIUS, DINAKAR RAMAKRISHNAN and V. S. VARADARAJAN

Formes modulaires sur la $\mathbb{Z}_{p}$-extension cyclotomique de $\mathbb{Q}$

LAURENT CLOZEL

Weight zero Eisenstein cohomology of Shimura varieties via Berkovich

275 spaces

MICHAEL HARRIS

$\Lambda$-adic Barsotti-Tate groups

HARUZO HIDA

Le flot géodésique des quotients géométriquement finis des géométries de

Hilbert

MickaËl CRAMPON and LUdOViC MARQuis

Nonplanarity of unit graphs and classification of the toroidal ones

A. K. Das, H. R. Maimani, M. R. Pournaki and S. YAssemi

Discrete semiclassical orthogonal polynomials of class one

DIEGO DOMINICI and FRANCISCO MARCELLÁN

A note on conformal Ricci flow

PENG LU, Jie QING and Yu Zheng

On representations of $\mathrm{GL}_{2 n}(F)$ with a symplectic period

ARNAB Mitra

Linked triples of quaternion algebras

ALEXANDER S. SIVATSKI

Finite nonsolvable groups with many distinct character degrees

Hung P. TONG-VIET

Errata to "Dynamics of asymptotically hyperbolic manifolds"

JULIE ROWLETT

Erratum to "Singularities of the projective dual variety" 